\title{
BIOMIMETICS OF TREE-SHAPED BRANCHING STRUCTURE IN TEXTILE FABRICS
}

\author{
J.T. FAN, M. SARKAR \& Q. CHEN \\ Institute of Textiles and Clothing, Hong Kong Polytechnic University, Hong Kong.
}

\section{ABSTRACT}

Water transport properties of fabrics are critical to wearing comfort. A tree-shaped branching network, which exists widely in nature, is known to have an extremely efficient transport system. The emulation of the treeshaped branching structure in textile fabrics has the potential of developing fabrics with superb liquid water transport properties for enhanced comfort. Here, we report on the development of some novel multilayer woven and knitted structures which mimic the branching structure. Experimental investigations have shown that the fabrics with such new structure have clearly faster liquid water transport and better moisture management property. These new fabrics are being commercialized.

Keywords: absorption, biomimetics, branching structure, fabrics, tree-shaped.

\section{INTRODUCTION}

Clothing is worn not only for basic protection and modesty but also for comfort. For active sportswear and clothing worn under hot and humid conditions, the liquid water transport properties of the constituent textile fabrics are critical in keeping the skin dry and promote comfortable well-being [1-4].

In recent years, there have been considerable research and developments in moisture management fabrics [5-10]. The term 'moisture management fabrics' can be defined as textile fabrics which provide the control of the movement of body water (e.g. sweat) and moisture in such a way that it is transported away from the skin to the outer surface of fabric where it can evaporate quickly. Garments produced from such fabrics keep the skin dry and provide maximum comfort to the wearer. Although conventional clothes made of pure cotton tend to have better sweat absorption properties, they tend to retain the sweat in the fabric layer next to the skin, giving the wearer an uncomfortable feeling of wet and cold after exercise. It thus becomes an objective of the field to develop fabrics which restrict the body sweat to spread in the inner layer (which touches body) and transport the sweat fast to the outer layer, where the sweat spreads as wide as possible so as to dry the cloth fast.

Moisture management fabrics were developed using different techniques, such as knitting, weaving or bonding. Majority of them were two or more layered fabrics produced by combining hydrophobic yarns in the inner layers, to keep the body dry, and hydrophilic yarns in outer layers [11-14], to absorb the body sweat through the inner layer. The main disadvantages of such fabrics are that the hydrophobic inner layer reduced the water absorption property of the fabric and may create discomfort sensation to our skin [15].

Trees are nature's creation for covering the earth. Trees not only possess the most important process for sustaining life on earth, i.e. photosynthesis, but also have an excellent water transport system, through which underground water can be transported against gravity from the roots all the way up to the leaves. Studies [16-18] have shown that the excellent water transport properties of trees are attributed to their unique branching network and the larger ratio of surface area to volume in the leaves than that in the stems of trees. It is therefore promising to emulate the tree structure in textile fabrics so as to enhance the water transport properties of the fabrics. Our previous research [19-22] demonstrated that a tree-shaped branching network can significantly improve the absorption property of various textiles. In this paper, we summarize the advantages of different types of 
tree-shaped branching fabrics against the standard interchanging fabrics, and compare this novel fabric with the standard market fabric.

\section{CONCEPT OF TREE-SHAPED BRANCHING STRUCTURED FABRIC}

Figures 1 and 2 illustrate the basic concept of three or two integrated layers tree-shaped branching fabrics. For three-layered tree-structured fabric (Fig. 1) the bottom layer is a 4/4 mock leno or matt structure, in which four yarns are grouped together to form a main 'stem'. The 'four-yarn stem' is then split into two 'two-yarn branches' in the middle layer, which can be a hopsack or matt. The top layer is a plain weave, in which the yarns are separate so as to have a greater surface area to volume ratio. In case of two layer tree-structured fabrics (Fig. 2), two yarns in the bottom layer of the fabric are grouped together and split into individual yarns by using plain design in the face of the fabric.
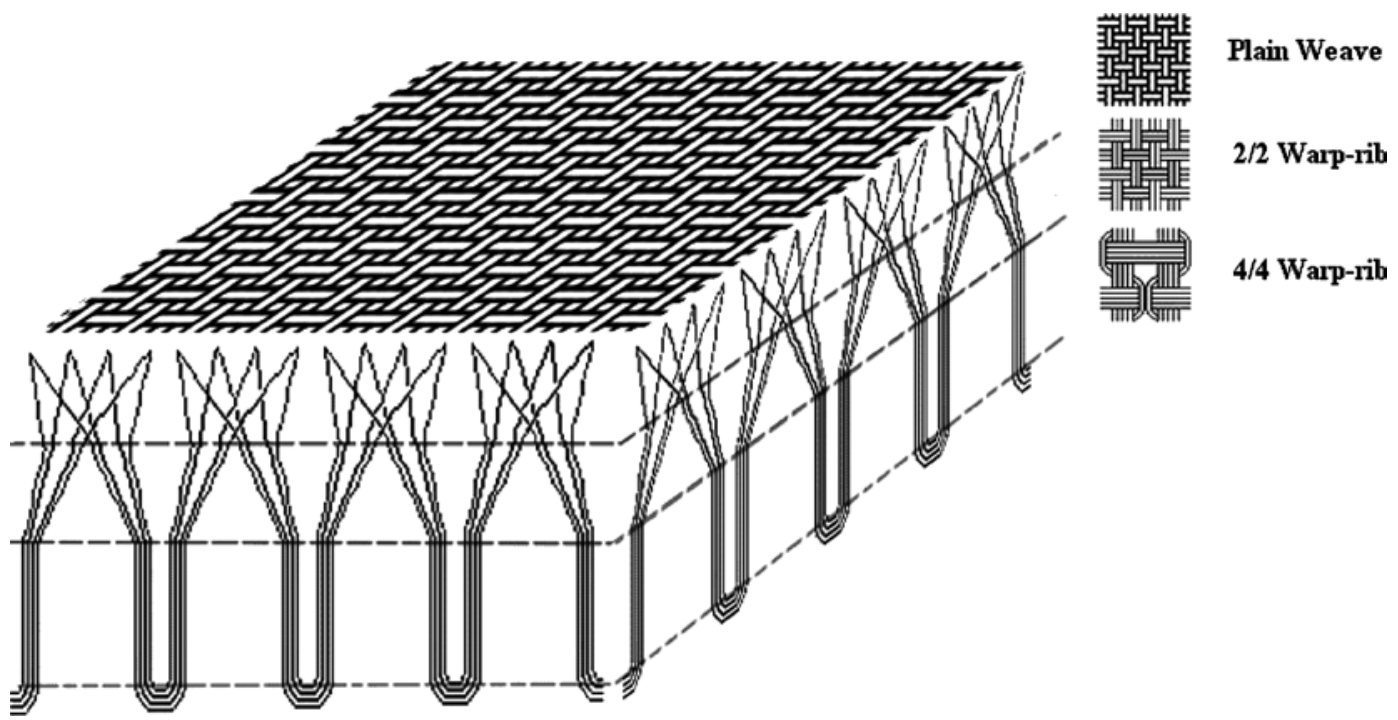

Figure 1: Concept of three-layer tree-shaped branching structured fabric.

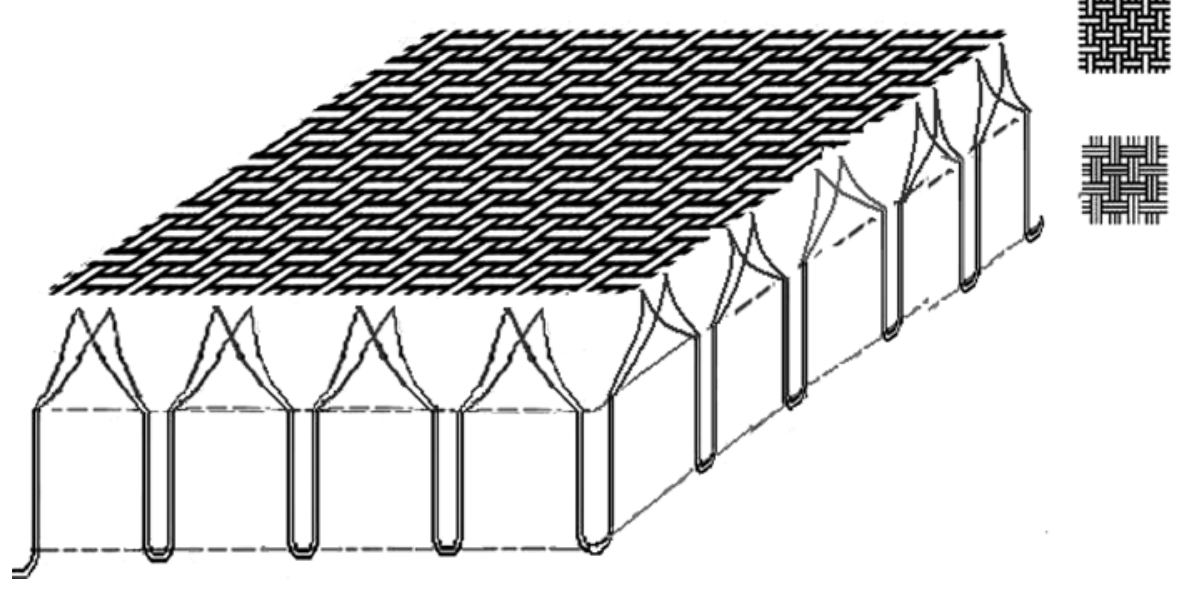

Plain Weave

Figure 2: Concept of two-layer tree-shaped branching structure fabric. 


\section{EXPERIMENTAL}

\subsection{Development of fabric samples}

Different three layers and two layers woven and knitted fabrics were developed to understand the advantages of tree-shaped branching fabrics. In this paper, we have considered six different fabrics to understand the advantages of branching structure. We also collected two standard woven fabrics (one plain weave and another twill weave) from the market to compare with our branching fabrics. The construction details of these fabrics are presented in Table 1. The details of multilayer fabrics are as follows:

1. Two three layer woven fabrics are considered. The first one was a three-layer branching structured (Sample no. 1) fabric with weft yarn interchanging as presented in Fig. 1, with 4/4 warp-rib design in the bottom-layer, 2/2 warp-rib design in the middle-layer and plain weave in the toplayer. The second one was a plain fabric with similar interchanging (Sample no. 2) pattern in the weft direction, developed for comparison.

2. Two two-layer $100 \%$ cotton woven fabrics are taken. One two-layer was tree-shaped branching woven fabric (Sample no. 4) was developed as per the conceptual structure as shown in Fig. 2, another was an interchanged plain fabrics (Sample no. 5) with same yarns were produced for comparison.

3. Two more two-layer tree-shaped branching knitted fabrics are considered with $100 \%$ cotton yarn. We used plating technique to achieve the two yarns together in the bottom layer, which opens completely in the face of the fabric as shown in the conceptual design.

\subsection{Testing of fabric samples}

All fabrics were tested in terms of constructional properties including thickness, thread density and mass. In addition, the liquid water transport properties, including the initial water absorption rate and the surface evaporation after saturation, were measured using the novel Transplanar Water Transport Tester (TWTT) [23, 24]. To characterize the moisture management property all fabrics were evaluated by the Moisture Management Tester (MMT) [25]. To better visualize the internal structure of the fabric, a microscopic picture was taken. An optical contact angle meter fitted with a high-speed camera was used to observe the absorption of water drop by the fabric.

\section{RESULTS AND DISCUSSION}

\subsection{TWTT testing results}

\subsubsection{Initial absorption}

The initial absorption rate is the absorption rate of the fabric sample when it first touches the water. The surface evaporation rate of the fabric is the amount of water evaporated per second from the fabric surface after it is completely saturated with water. The initial absorption of the fabric is calculated as the mean rate of absorption in the initial 5 seconds. The average absorption rate and mean surface evaporation rate were taken based on the three individual tests of each fabric, all results are presented in Table 2. To understand the differences between different fabric samples, $t$-tests were conducted.

Figure 3 compares the absorption of liquid water (indicated by the reduction in water in TWTT) against time. The sharp fall of the initial curve in the figure indicates that the initial absorption of the 


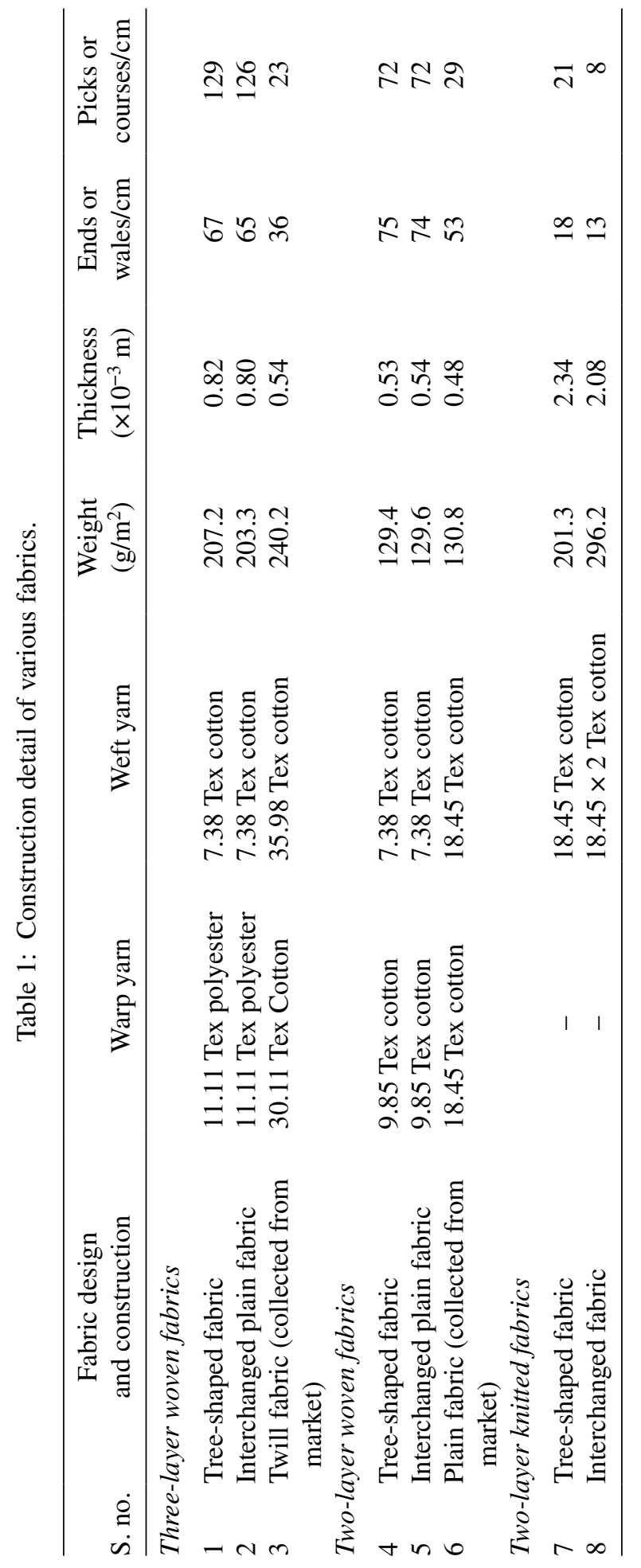


Table 2: Absorption results of different fabrics.

\begin{tabular}{|c|c|c|c|c|c|c|}
\hline S. no. & Fabric design and construction & $\begin{array}{c}\text { Initial } \\
\text { absorption } \\
(\mathrm{g} / \mathrm{sec})\end{array}$ & $\mathrm{CV}(\%)$ & $\begin{array}{c}\text { Surface } \\
\text { evaporation } \\
(\mathrm{g} / \mathrm{sec})\end{array}$ & $\mathrm{CV}(\%)$ & $\begin{array}{l}\text { One way } \\
\text { water } \\
\text { transport }\end{array}$ \\
\hline \multicolumn{7}{|c|}{ Three-layer woven fabrics } \\
\hline 1 & $\begin{array}{l}\text { Thee-layer branching-structure } \\
\text { fabric }\end{array}$ & 1.94 & 2.88 & 0.0063 & 2.12 & 170.52 \\
\hline 2 & Three-layer interchanged plain & 1.75 & 2.68 & 0.0061 & 2.01 & 99.42 \\
\hline 3 & $\begin{array}{l}\text { Twill fabric (collected from } \\
\text { market) }\end{array}$ & 1.44 & 3.41 & 0.0065 & 2.82 & 95.28 \\
\hline \multicolumn{7}{|c|}{ Two-layer woven fabrics } \\
\hline 4 & $\begin{array}{l}\text { Two-layer branching-structure } \\
\text { fabric }\end{array}$ & 1.82 & 2.06 & 0.0065 & 2.89 & 128.31 \\
\hline 5 & Interchanged plain & 1.72 & 2.95 & 0.0065 & 2.67 & 97.86 \\
\hline 6 & $\begin{array}{l}\text { Plain fabric (collected from } \\
\text { market) }\end{array}$ & 1.62 & 2.35 & 0.0063 & 2.32 & 76.28 \\
\hline \multicolumn{7}{|c|}{ Two-layer knitted fabrics } \\
\hline 7 & $\begin{array}{l}\text { Two-layer branching-structure } \\
\text { fabric }\end{array}$ & 2.34 & 2.12 & 0.0073 & 2.73 & 15.79 \\
\hline 8 & Interchanged plain & 2.08 & 1.50 & 0.0068 & 2.64 & -39.71 \\
\hline
\end{tabular}

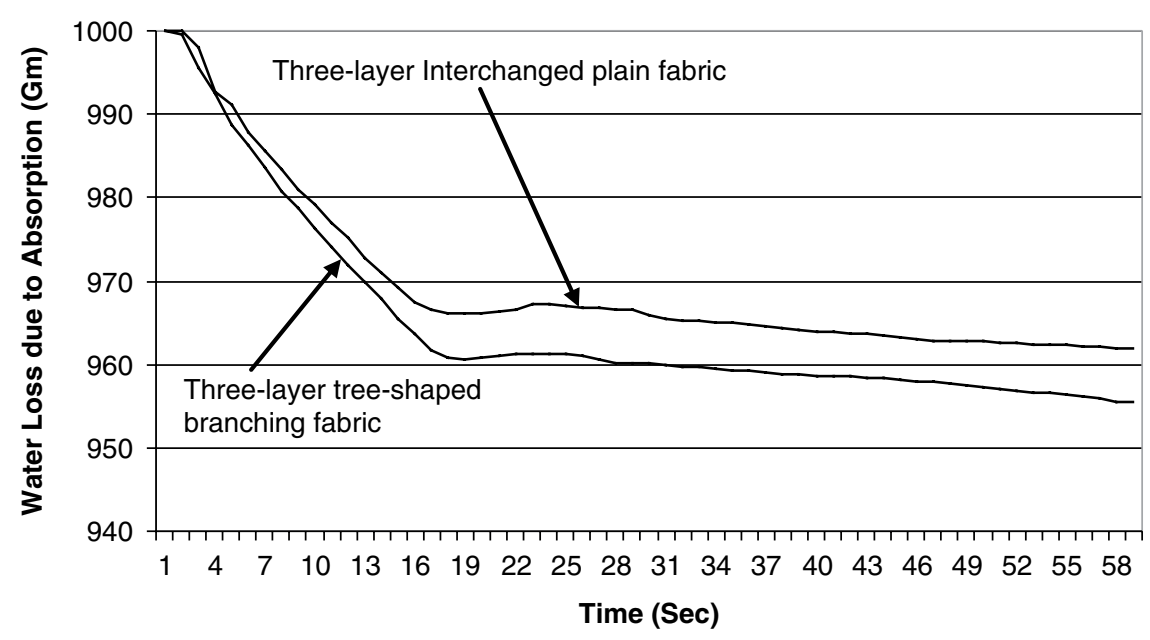

Figure 3: Comparison of water loss due to initial absorption by the different three-layer fabrics.

tree-shaped branching fabric (Sample no. 1) is approximately $11 \%$ faster $(p$-value $<0.01)$ than the corresponding three-layer interchanging plain fabric (Sample no. 2). Results also indicated that three-layer branching fabric absorbed significantly quicker as compared to market twill fabric with similar weight (Sample no. 3).

Similar trend can be observed for two-layer fabrics. Figure 4 indicates tree-shaped branching woven and knitted fabrics (Sample nos 4 and 7) have significant faster water absorption (5.8\% and 


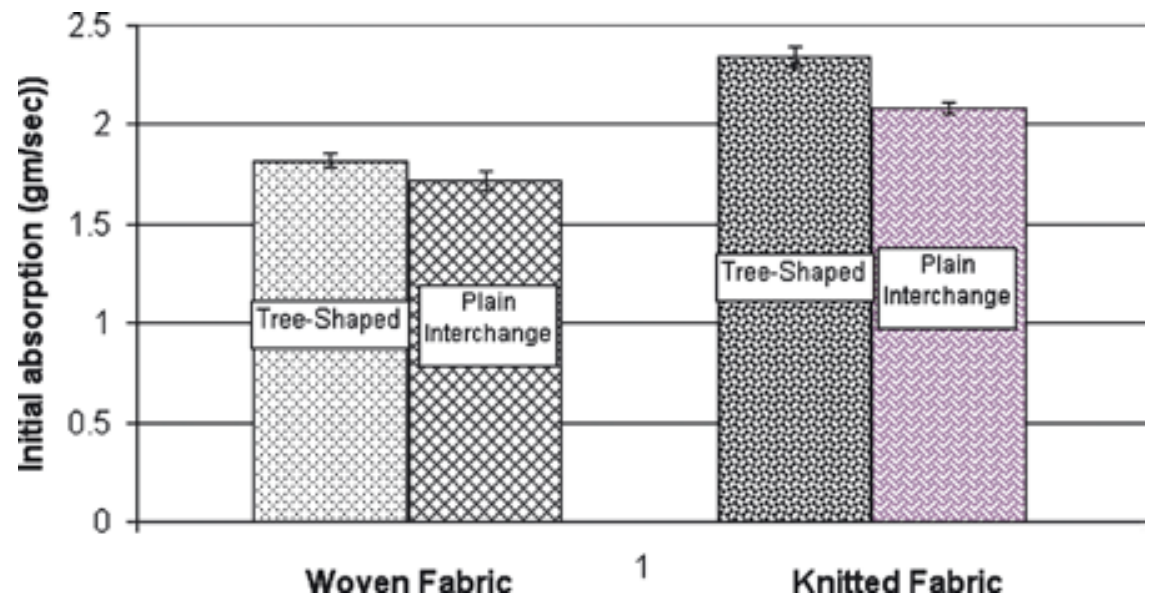

Figure 4: Initial absorption of different two-layer fabrics.

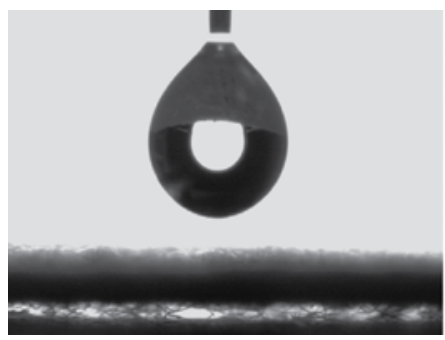

(a)

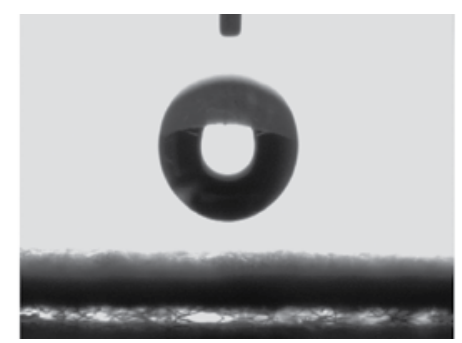

(b)

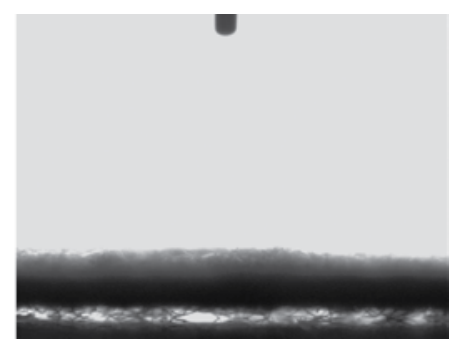

(c)

Figure 5: Absorption of water-drop by two-layer cotton branching structured fabric (time between two shots is 21.4 milliseconds or 0.021 seconds).

$12.5 \%$, respectively) rate than the corresponding interchange plain fabrics (Sample nos 5 and 8 , respectively). When the woven branch fabric (Sample no. 4) was compared with standard plain fabric (Sample no. 6) of similar weight collected from the market, a significant $12.3 \%$ faster absorption was observed for the former one.

\subsubsection{Surface evaporation}

Surface evaporation rates were also measured on the TWTT when the fabrics are completely saturated with water. Table 2 lists the average values of surface evaporation rate over four hours for different types of fabrics. The values indicate only marginal differences among the fabrics. Such differences are not significant. This is understandable, as the fabrics have similar surface characteristics, the evaporation from these surfaces saturated with water should be similar.

\subsection{Image of water absorption by high-speed camera}

To visualize the absorption of the water by the fabric surface, we took a picture using high-speed camera of the OCA tester, depicted in Fig. 5a-c. The picture shows that the water vanished on the fabric surface of a $100 \%$ cotton two-layer branching structured fabric in less than 0.021 seconds. 


\subsection{MMT results}

The one-way transport value was measured by the MMT tester, which indicates the movement of water from the inner side to the outer side of the fabric, i.e. the movement of liquid in a transplanar direction. For all tree-shaped branching structured fabrics, a quicker one-way transport behavior was observed as compared to interchanging plain fabrics with similar yarns and weight. Three-layer branching structured fabric (Sample no. 1) shows approximately $42 \%$ benefit over the similarly interchanged plain fabric (Sample no. 2). A similar trend was observed for the two-layer woven and knitted fabrics. Two-layer woven branching fabric also had faster water transport in the transplanar direction against the market plain fabric. This indicated that plant-structure fabric quickly transports the body water (sweat) to the outer layer and keeps the skin dry.

\subsection{Possible mechanism}

The superior liquid water transport properties of fabrics emulating the branching structure of trees may be a result of a combination of different mechanisms. Figure 6 illustrates the magnified view of the face and back of the two-layer plant structured cotton fabric. The picture shows that in the back of the fabric two yarns are united together as described earlier, which open-up into individuals on the face of the fabric. As the density of the yarns is equal on both sides of the fabric, a bigger air-space of the back of the fabric reduced toward the face side, which creates a pull of capillary pressure [26] towards the toplayer. For the three-layer fabrics, a similar phenomenon was noticed. In addition, same yarns of the bottom move to the top-layer facilitating transportation of water from the bottom to the top layer, which may be enhanced further due to the branching nature [26] of the tree-like structure.

\section{CONCLUSION}

This study revealed that tree-shaped two-layer and three-layer branching structure can enhance the absorption property of the textile fabric in the in-plane and trans-plane direction. The two-layer light weight plant structured fabric has good potential to be used as dress shirt materials in the tropical atmosphere. Due to high absorption property, branching structured knitted fabrics have the potential to be used for the sportswear. The most important aspect of the plant structured fabrics is that, the superior moisture management property of the plant structured fabrics is based on a special structure, so it remains unaffected by repeated washing.

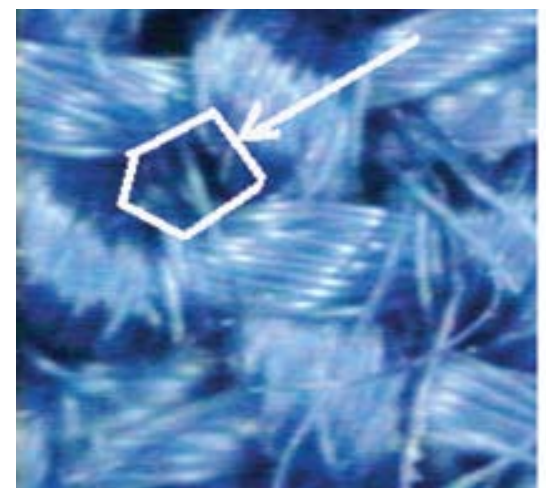

(a)
Face of the fabric

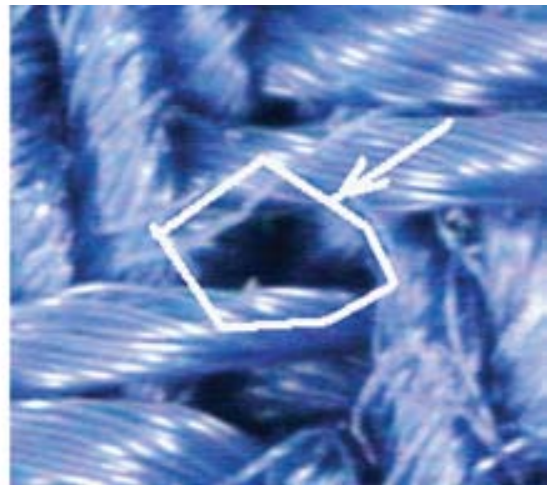

(b)
Back of the fabric

Figure 6: Magnified view of the two-layer plant structured fabric. 


\section{ACKNOWLEDGEMENTS}

The authors would like to acknowledge the funding support of the Research Grant Council of HKSAR (Grant No.: PolyU 5164/06E), the Innovation \& Technology Commission of HKSAR (ITSF Project: ITP/021/08TI) and the Hong Kong Polytechnic University (Niche Area Project: $1-\mathrm{BB} 82)$.

\section{REFERENCES}

[1] Gagge, A.P. \& Gonzalez, R.R., Physiological and physical factors associated with warm discomfort and sedentary man. Environ. Res., 7, p. 230, 1974. doi:10.1016/0013-9351(74)90154-6

[2] Scheurell, D.M., Spivak, S.M. \& Hollies, N.R.S., Dynamic surface wetness of fabrics in relation to clothing comfort. Text. Res. J., 55, p. 394, 1985. doi:10.1177/004051758505500702

[3] Lau, L., Fan, J., Siu, T. \& Siu, L.T.C., Comfort Sensations of polo shirts with and without wrinkle free treatment, Text. Res. J., 72(11), pp. 949-953, 2002. doi:10.1177/004051750207201103

[4] Chen, Y.S., Fan, J. \& Zhang, W., Comfort sensation of polo shirt with or without wrinkle free treatment. Text. Res. J., 73, p. 152, 2003. doi:10.1177/004051750307300210

[5] Riegger, S., Weft binding layered knitting, US Patent No. 6089052, 1998.

[6] Kasdan, R. \& Kornblum, S., Synthetic knit fabric having superior wicking and moisture management properties, US Patent No. 6427493 B1, 2002.

[7] DeMott, R.P., Gillis, K.C. \& Stringer, E.G., Absorbent fabrics, products, and methods, US Patent No. 6770581, 2004.

[8] Lee, J.H., Kim, S.H., Lee, K.J., Lim, D.Y. \& Jeon, H.Y., Determining the absorption properties of split-type microfiber fabrics by measuring the change in color depth. Text. Res. J., 74, p. 271, 2004. doi:10.1177/004051750407400315

[9] Rodrigues, K.A., Hydrophilic finish for textiles, US Patent No. 6046120, 2000.

[10] Ferrero, F., Wettability measurement of plasma treated synthetic fabrics by capillary rise method. Polym. Test., 22, p. 571, 2003.

[11] Hozuma, O., Sweat-absorbent textile fabric, US Patent No. 4530873, 1985.

[12] Strauss, I. \& Rankin, S.A., Fabric for recreational clothing, US Patent No. 5050406, 1991.

[13] Lee, Y.K., Method for making fabric with excellent water transition ability, US Patent No. 6381994, 2002.

[14] Yeh, P., Fabric for moisture management, US Patent No. 6509285, 2003.

[15] Ha, M., Tokura, H. \& Yanai, Y., Combined effects of fabric air permeability and moisture absorption on clothing microclimate and subjective sensation during intermittent exercise at 27 degrees C. Ergonomics, 42, p. 964, 1999.

[16] Daum, C.R., A method for determining water transport in trees. Ecology, 48, p. 425, 1967.

[17] Koch, G.W., Sillett, S.C., Jennings, G.M. \& Davis, S.D., The limits of tree height. Nature, 428, p. 851, 2004.

[18] Stern, K.R., Jansky, S. \& Bidlak, J.E., Introductory Plant Biology, McGraw Hill: New York, 2003.

[19] Fan, J.T., Sarkar, M.K., Szeto, Y.C. \& Tao, X.M., Plant structured textile fabrics. Materials Letters, 61(2), p. 561, 2007.

[20] Fan, J.T., Sarkar, M.K., Szeto, Y.C. \& Tao, X.M., Biomimetics of plant structure in textile fabrics for the improvement of water transport properties. Tex. Res. J., 79, p. 657, 2009.

[21] Fan, J.T., Sarkar, M.K., Szeto, Y.C. \& Tao, X.M., Development and characterization of light weight plant structured fabrics. Fibers \& Polymers, 10, p. 343, 2009. 
[22] Chen, Q., Fan, J.T., Sarkar, M.K. \& Jiang, G.M., Biomimetics of plant structure in knitted fabrics to improve the liquid water transport properties. Text. Res. J., 80(6), pp. 568-576, 2010. doi: $10.1177 / 0040517509340600$

[23] Fan, J.T., Sarkar, M.K. \& Qian, X.M., Transplanar water transport tester for fabric. Measurement Science and Technology, 18, p. 1465, 2007. doi:10.1088/0957-0233/18/5/036

[24] Sarkar, M.K., Fan, J.T. \& Chen, Q., Transplanar water transport tester for fabrics, The 86th Textile Instituted World Conference. Hong Kong, 18-21 Nov 2008.

[25] Yao, B.G., Li, Y., Hu, J., Kowok, Y.L. \& Yeung, K.K., An improved test method for characterizing the dynamic liquid moisture transfer in porous polymeric material. Polym. Test., 25, p. 677 , 2006. doi: $10.1177 / 004051750507500111$

[26] Hu, J., Li, Y., Kwok-Wing, Y., Wong Anthony, S.W. \& Xu, W., Moisture management tester: a method to characterize fabric liquid moisture management properties. Text. Res. J., 75, p. 57, 2005.

[27] Bejan, A., Shape and Structure - from Engineering to Nature, Cambridge University Press: Cambridge, UK, pp. 78-108, 2000. 